\title{
Estudio de los fundentes utilizados en la metalurgia del cobre en Cerro Muriano (Córdoba) durante el período Romano Altoimperial $^{(\bullet)}$
}

\begin{abstract}
A.J. Criado $^{(*)}$, R. Calabrés ${ }^{(*)}$, J.A. Martínez ${ }^{(*)}$ y J.J. Storch de Gracia ${ }^{(* *)}$
Resumen Durante el Imperio Romano, de todos los distritos mineros explotados por Roma -Hispania, Galia, las provincias danubianas, Dacia, Grecia, Asia Menor, Siria, Egipto y el Norte de África-, sin duda el más rentable y más abundante en recursos era Hispania. Plinio facilita muchos datos en los pasajes dedicados a la minería y el trabajo de los metales en su Historia Natural. Entre los muchos lugares mineros que este autor cita, nos interesa ahora la explotación de cobre en cerro Muriano, del cual dice que era de una calidad inmejorable, utilizado, entre otras aplicaciones, para fabricar el famosísimo cobre de Campania. Para poder obtener esta elevada calidad, es fundamental la utilización de fundentes adecuados en el proceso de obtención de cobre. La adición de estos fundentes era vital para controlar los parámetros de trabajo y alcanzar las temperaturas requeridas. La elevada pureza del cobre obtenido demuestra un conocimiento muy ajustado de los fenómenos que tenían lugar.
\end{abstract}

Palabras clave: Cobre. Fundentes. Metalografía. Fundición. Siglo I d.C.

\section{Study of the fusing agents used in the metallurgy of copper at Cerro Muriano (Cordoba) during the High-Imperial Roman period}

\begin{abstract}
There are no doubts that Hispania was, under the Roman Empire, the most profitable and abundant in resources of all the mining districts exploded by Rome -Hispania, Galia, Danubian Provinces, Dacia, Greece, Asia Minor, Syria, Egypt and North Africa-. Plinius gives the same data and hands many more in the passages dedicated to the mining and the working of the metals in his Natural History. Of all the many mining places that this author refers, the exploiting in copper at Cerro Muriano presents the main interest; of this he says that it was of an unbeatable quality, used, among other applications, to manufacture the very famous "Copper of Campania". The utilization of fusing agents, adapted to the process of copper obtainment, is fundamental for obtaining this high quality. The addition of these fusing agents was very important for the control of the work parameters and to reach the required temperatures. The high purity of the obtained copper shows a very close knowledge of the phenomena involved in the process.
\end{abstract}

Keywords: Copper. Fusing agents. Metallography. Smelting. 1st Century A.C.

\section{INTRODUCCIÓN}

Desde los primeros momentos de la metalurgia extractiva del cobre durante el Período Calcolítico, la utilización de fundentes ha sido práctica habitual,

(•) Trabajo recibido el día 17 de noviembre de 1995.

(*) Dpto. de Ciencia de los Materiales. Facultad de Ciencias Químicas. Universidad Complutense de Madrid. 28040Madrid (España).

(**) Dpto. de Ciencias y Técnicas Historiográficas. Facultad de Geografía e Historia. Universidad Complutense de Madrid. 28040-Madrid (España). más o menos intencionada o casual, adaptados siempre a la composición química de las rocas y minerales empleados (1-3). La necesidad de alcanzar mayores temperaturas aumentando el rendimiento del horno metalúrgico con los combustibles utilizados, de escaso aporte calorífico, de evitar la oxidación del metal naciente fundido y de eliminar impurezas del metal hizo necesaria la utilización de fundentes de diferente naturaleza.

La calidad del cobre obtenido dependía de la composición de los minerales seleccionados (calcopiritas en el caso del período romano), fundentes empleados, tipo de combustible, diseño y utiliza- 
ción de los hornos, control de la temperatura y soplado de aire. Durante el Imperio Romano se hizo célebre el cobre Mariano -aes marianum según Plinio, por ser Sexto Mario su propietario- (4-6) por su bondad y por ser la materia prima para fabricar el famosísimo cobre de Campania, aleándolo, a su vez, con plomo de Hispania, para solventar los condicionantes anteriormente citados. Este cobre Mariano se fabricaba en las instalaciones minero metalúrgicas de Cerro Muriano situado a $16 \mathrm{~km}$ al Norte de la ciudad de Córdoba, capital de la Bética (Fig. 1) (7 y 8).

Las muestras de escorias y fundentes se recogieron en el yacimiento arqueológico de Siete Cuevas, en un escorial correspondiente al siglo I d.C. según indicaron los restos de cerámicas encontrados. Se han elegido aquellos ejemplares que proporcionaron la información más clara: escorias poco fundidas que contenían clastos -partículas residuales, o aglomerados de partículas del fundente inicial, que no llegaron a fundirse y que se encuentran rodeados de material fundido denominado matriz- de los diferentes fundentes y del mineral empleado envueltos por un cemento fayalítico correspondiente a los materiales que llegaron a reaccionar y fundir (Fig. 2) y trozos de materiales añadidos como fundentes y que sólo reaccionaron parcialmente (Fig. 3). Todos estos materiales aportan una información valiosa sobre su utilización al presentar, en estadios intermedios, el proceso seguido que ha quedado inacabado a causa de su situación en el horno bien por fallos en el diseño o manipulación de éste.

\section{TÉCNICA EXPERIMENTAL}

Las muestras fueron recogidas y seleccionadas en el escorial situado a doscientos metros al Oeste de la mina romana de Siete Cuevas, junto a la zona

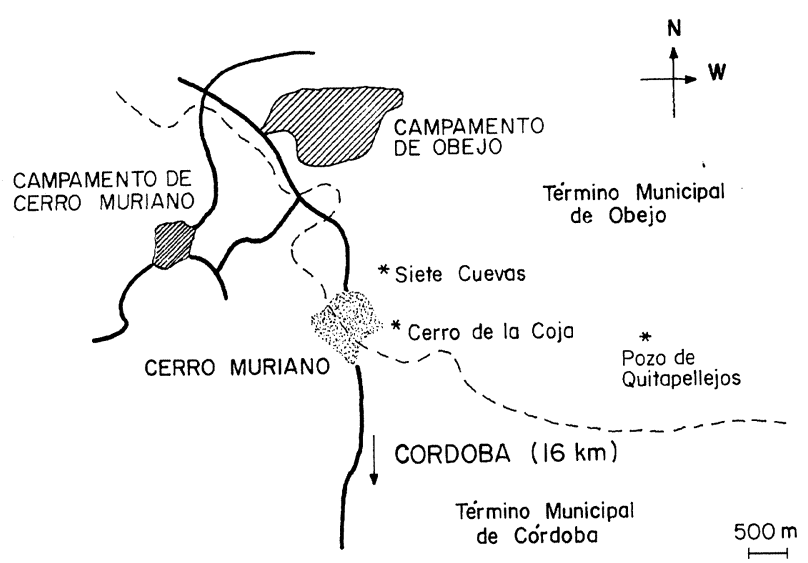

FIG. 1.- Mapa esquemático de la situación geográfica de Cerro Muriano (Córdoba).

\section{FIG. 1.- Schematic map of the geographical} situation of Cerro Muriano (Córdoba).

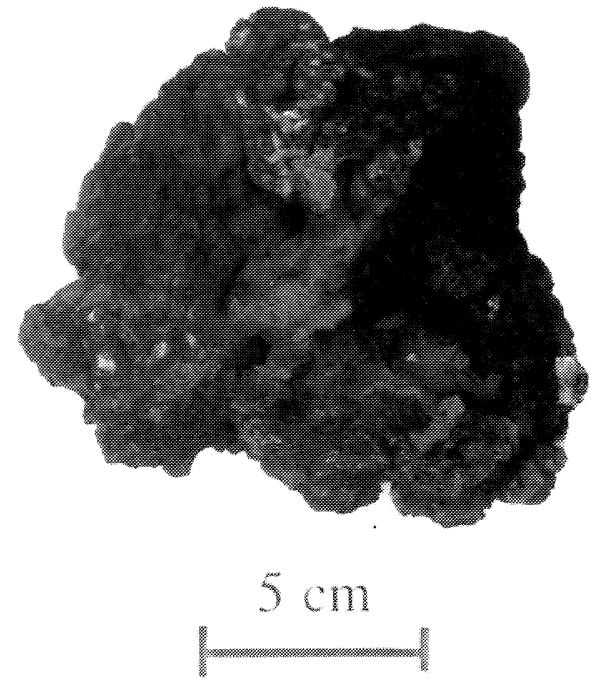

FIG. 2.- Escorias escasamente fundidas mostrando parte de los componentes originales intactos.

FIG. 2.- Scarcely smelted slags showing a part of the intact original components.

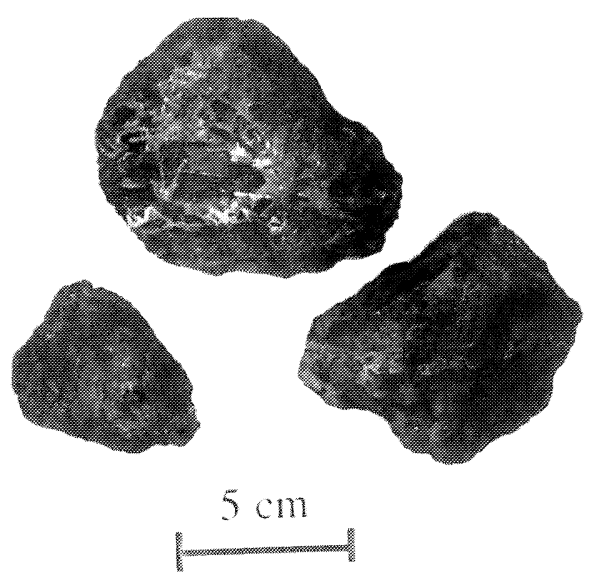

FIG. 3.- Trozos de mineral de hierro (hematites) que ha reaccionado parcialmente con el cuarzo, añadidos como fundentes.

FIG. 3.- Pieces of iron ore partially reacted with the quartz added as fusing agents.

de hornos de fundición situada en la actual acera del Cuartel Viejo en Cerro Muriano (Fig. 1).

Las piezas seleccionadas se trocearon para obtener superficies de fractura limpias que pudieran facilitar información. Después de sometidas a limpieza y sputtering de oro -con el fin de hacerlas conductoras- se observaron mediante microscopía electrónica de barrido (SEM) y se analizaron mediante la técnica de dispersión de energías (EDS).

También se prepararon muestras para difracción de rayos X (DRX) y superficies planas de forma convencional para realizar el estudio metalográfico. En los casos en que fue necesario ataque químico se 
utilizó un reactivo a base de HF en solución acuosa al $45 \%$ en masa durante 2 min.

\section{RESULTADOS Y DISCUSIÓN}

Durante las operaciones de reducción en el horno metalúrgico, después de la operación de tostación de la calcopirita, el funcionamiento de éste no es homogéneo. Es difícil, incluso en la actualidad, conseguir una distribución correcta de la carga mineral (tostado), fundentes y combustible (carbón de leña), y más problemático aún conseguir que el soplado sea el necesario para no enfriar la carga y sí quemar el combustible para alcanzar la temperatura y el ambiente reductor exigido. La distribución y tamaño de los componentes de la carga significan un gran obstáculo para la circulación del aire y de los gases, con el problema añadido de las modificaciones severas sufridas por dicha carga durante las transformaciones físicas, químicas y mecánicas que se producen durante el proceso. Todo ésto conlleva a que las operaciones del horno no sean homogéneas y haya zonas en las que las reacciones sean incompletas o no se lleven a cabo por la no conjunción de las variables antes descritas. Así, en la descarga final y limpieza del horno se pueden extraer materiales inalterados o parcialmente procesados.

En esta investigación se han seleccionado muestras de materiales que no han sufrido una conversión total en el horno por lo que presentan características singulares tales como materiales sin procesar y otros en fase de transformación (Figs. 2 y 3). De esta forma se puede aclarar la naturaleza de los fundentes utilizados en la metalurgia romana del cobre en Cerro Muriano.

En la figura 4 se observa una escoria poco fundida, parcialmente procesada, mostrando constituyentes de óxido de hierro (hematites) (A) y tectosilicatos (grupo de las zeolitas) sin reaccionar (B). Son clastos de diferente naturaleza mineral unidos por un cemento de silicato de hierro (fayalita) (C) que es quien cohesiona el conglomerado heterogéneo y que se corresponde con la minoritaria cantidad de materiales que han tenido suficiente tiempo y temperatura para reaccionar y fundir. Todo este conjunto tiene un aspecto granuloso típico que diferencia perfectamente las escorias fundidas de las no fundidas (Fig. 2), o sea las que alcanzan la temperatura necesaria y las que no lo consiguen (2, 3 y 9 ).

La no alteración de estos materiales fundentes añadidos al horno permite identificar la mezcla usada: arenas de sílice más o menos impurificadas con tectosilicatos del grupo de las zeolitas (Figs. 5 y 6), calcita y baritina y óxidos de hierro, principalmente hematite (oligisto), en masas aglomeradas terrosas de color rojo cereza intenso sobre una matriz alveolar de cuarzo, raras veces cálcica (calcita). En ocasiones, el oligisto se ha comprobado presentado a magnetita y a limonita, aunque siempre está asociado en una matriz silícea o cálcica.

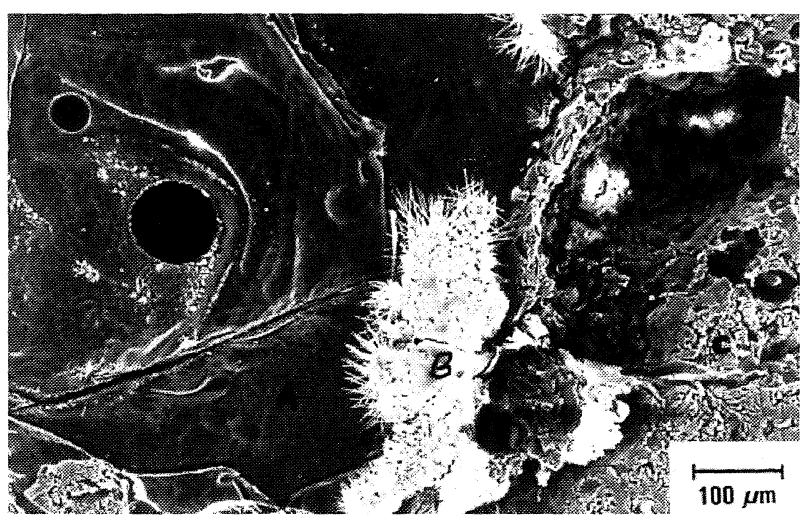

FIG. 4.- Escoria poco fundida mostrando parte de los componentes originales del fundente sin reaccionar: (A) hematites, (B) silicatos complejos y (C) cemento de fayalita (silicato de hierro).

FIG. 4.- Scarcely smelted slag showing a part of the original components of the no reacted fusing agent: (A) hematine iron ore, $(B)$ complex silicates and $(C)$ cement of fayalite (iron silicate).

La proporción de óxidos de hierro en el fundente está subordinada a conseguir una fayalita $(2 \mathrm{FeO}-$ $\mathrm{SiO}_{2}$ ) (9) de contenido adecuado en óxido de hierro con el fin de conseguir la máxima fluidez y el mínimo punto de fusión $(2,3$ y 9). Hay que pensar que durante el proceso de obtención del cobre a partir de la calcopirita, con previo proceso de tostación, la mayor parte del hierro en forma de óxido se va incorporando a la escoria sobrenadante con lo que su contenido en este metal aumenta, lo que puede incidir negativamente en las características físicas y mecánicas de la escoria fayalítica.

La presencia de tectosilicatos (zeolitas) aportaba fluidez a la escoria, lo que hace suponer que no

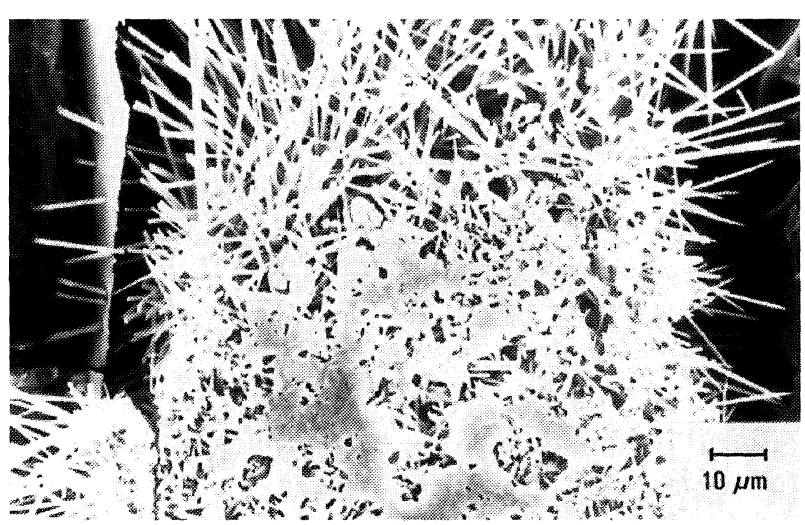

FIG. 5.- Estructura filamentosa de tectosilicato (del grupo zeolitas) sin reaccionar en la matriz cementante granulosa de fayalita.

FIG. 5.- Filamentous structure of no reated tectosilicate (of zeolites group) in the cemented granular matrix of fayalite. 


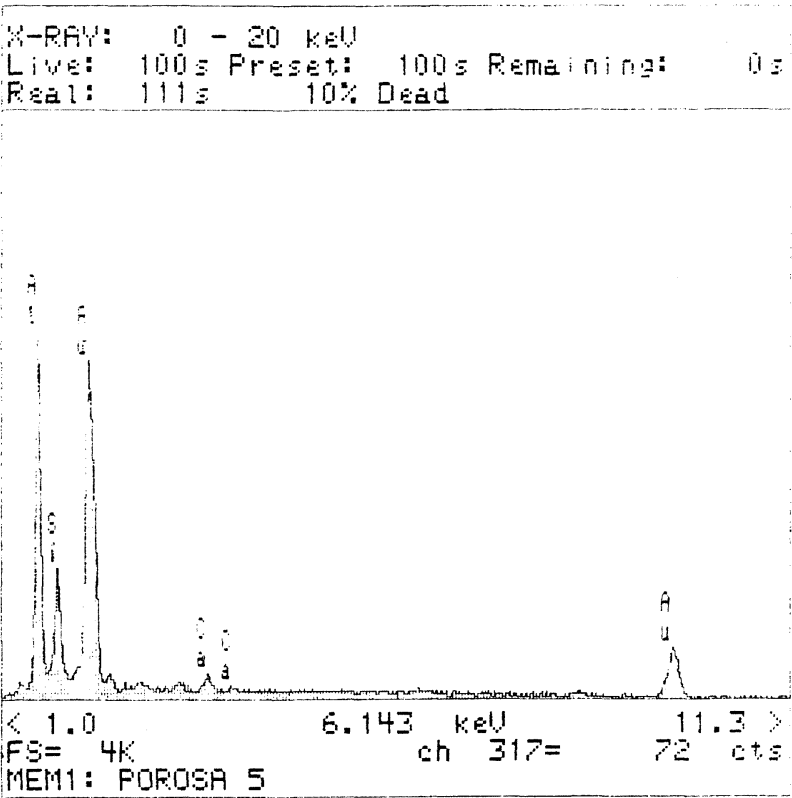

FIG. 6.- Análisis mediante EDS de la estructura filamentosa de la figura 5. La aparición del pico correspondiente al oro se debe al sputtering realizado en la muestra.

FIG. 6.- Identification by EDS of the filamentous structure of the figure 5. The appearance of the pick corresponding with gold is due to the sputtering made to the sample.

eran añadidos como ganga o impurezas sin ninguna razón, sino como algo tendente a fluidificarla.

Gracias a materiales incompletamente procesados, como el de la figura 3 , se ha podido sorprender el proceso de reacción entre el cuarzo y el óxido de hierro al alcanzar la temperatura adecuada.

En las figuras 7 y 8 se muestran oolitos de silicato de hierro formados en capas. sucesivas por reacción del silicato rico en cuarzo con el óxido de hierro (oligisto) que forma concreciones en ésta (Figs. 9 y 10). La fractura ha permitido ver la reacción y las capas sucesivas formadas. También se pueden observar capas ricas en $\mathrm{SiO}_{2}$ sobre el óxido de hierro (Fig. 11). Si hubiese transcurrido tiempo suficiente, la reacción hubiera sido total y se habría formado la fayalita correspondiente.

El interés de esta reacción está en la baja temperatura de fusión del silicato de hierro formado (10) y en la posibilidad de que la sílice añadida elimine el óxido de hierro, no sólo el adicionado como fundente, sino también el procedente de la oxidación de la mata. De esta forma era posible rebajar el contenido de hierro de la mata a valores insignificantes, cuestión vital para conseguir un cobre de primera fusión de alta calidad y pureza.

La formación de fayalita de bajo punto de fusión y baja viscosidad a partir de la reacción del cuarzo, tanto con el óxido de hierro mineral (oligisto) como con el formado durante el soplado y oxidación de la

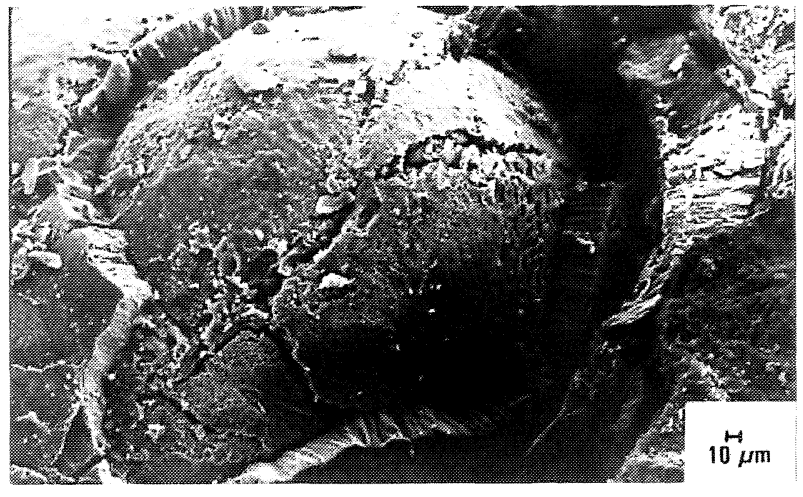

FIG. 7.- Oolitos de silicato de hierro (fayalita) en capas concéntricas, originadas por reacción de la hematites terrosa en forma de concreciones con el silicato al alcanzar el horno la temperatura adecuada.

FIG. 7.- Oolites of iron silicate (fayalite) in concentric caps, originated by reaction of the hematine iron ore concretioned with the silicate. This compounds are produced in the oven at the adequate temperature.

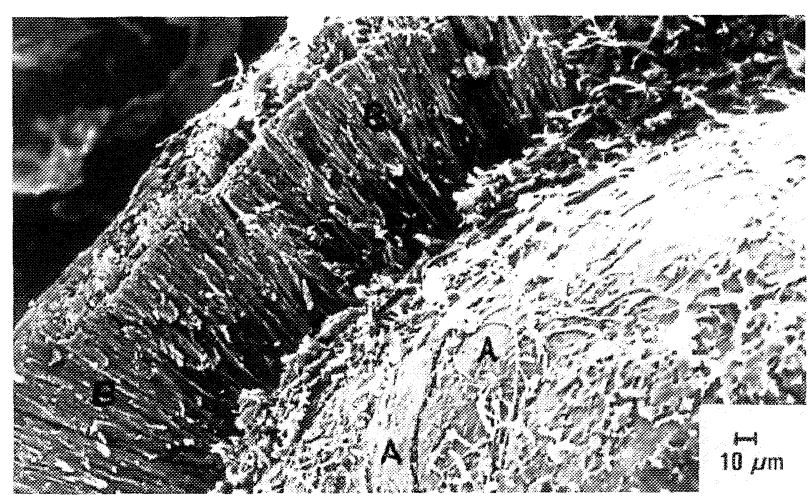

FIG. 8.- Detalle a mayores aumentos de los oolitos de fayalita de la figura 7 .

FIG. 8.- High magnification detail of the oolites of fayalite of the figure 7.

mata, fue la razón principal para utilizar estos materiales como fundentes en la metalurgia del cobre en Cerro Muriano durante el Imperio Romano.

\section{CONCLUSIONES}

- Los materiales arqueológicos seleccionados han permitido observar y estudiar la naturaleza del fundente utilizado en la metalurgia extractiva del cobre en Cerro Muriano durante el Imperio Romano, constituido fundamentalmente a base de arena de cuarzo y óxido de hierro (hematites).

- Las superficies de fractura y las preparadas por metalografía convencional, atacadas químicamente cuando el ataque fue requerido con $\mathrm{HF} 45$ $\%$ durante $2 \mathrm{~min}$, han permitido observar correc- 


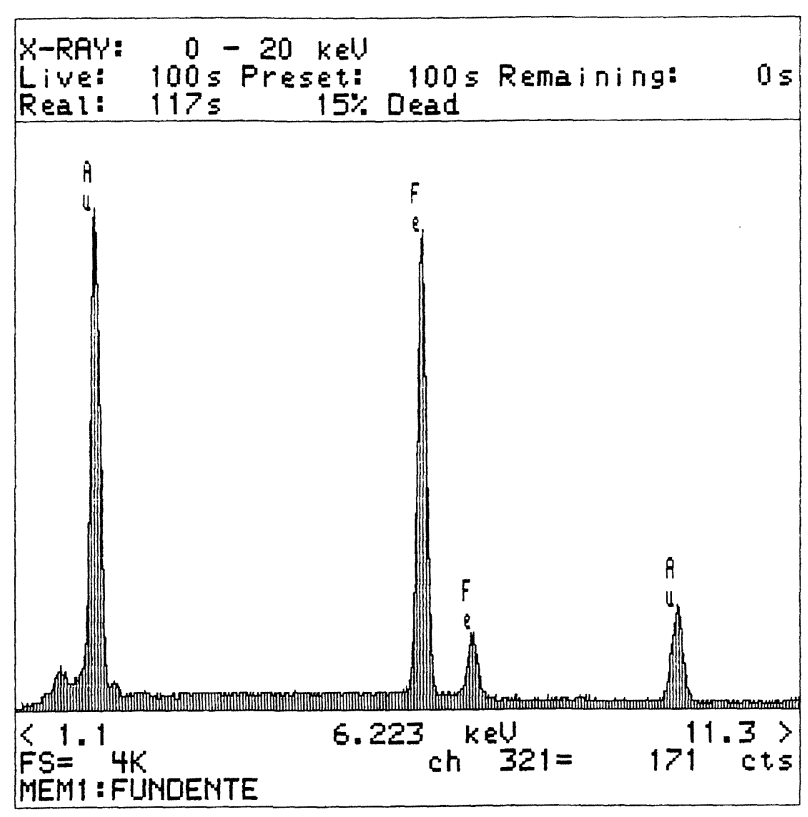

FIG. 9.- Análisis mediante EDS de la zona de oligisto sin reaccionar con el silicato de la figura 8 (A).

FIG. 9.- Identification by EDS of the zone of no reacted oligist from the figure $8(A)$.

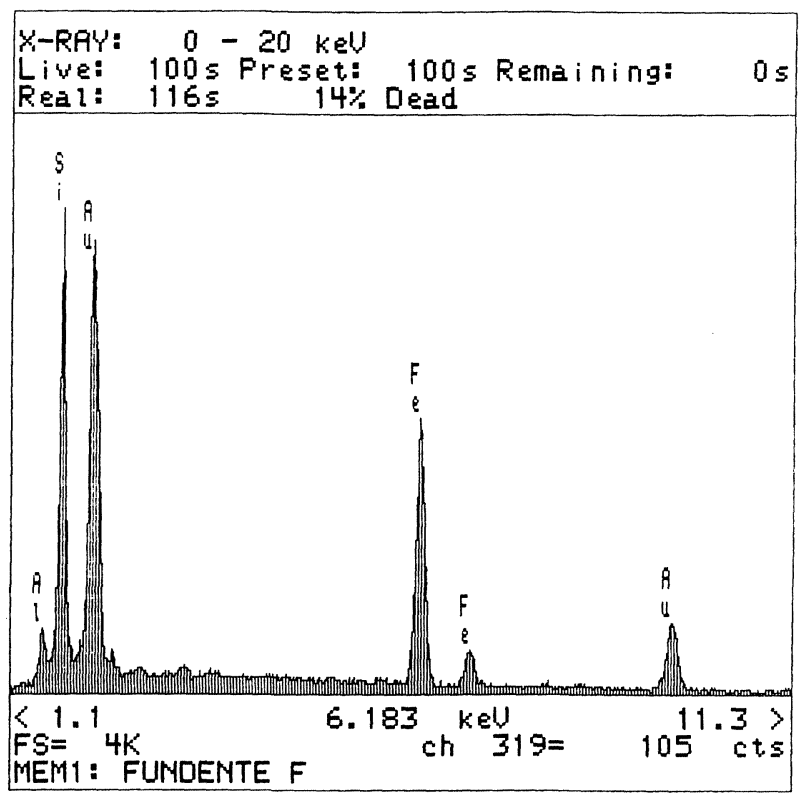

FIG. 10.- Análisis mediante EDS de los oolitos de fayalita de la figura $8(\mathrm{~B})$.

FIG. 10.- Identification by EDS of the oolites of fayalite of the figure $8(B)$.

tamente, mediante el SEM, la estructura de los fundentes utilizados.

- La utilización de técnicas como EDS y DRX han servido para identificar los diversos componentes que conformaban los fundentes utilizados, en algunos casos de naturaleza muy compleja.

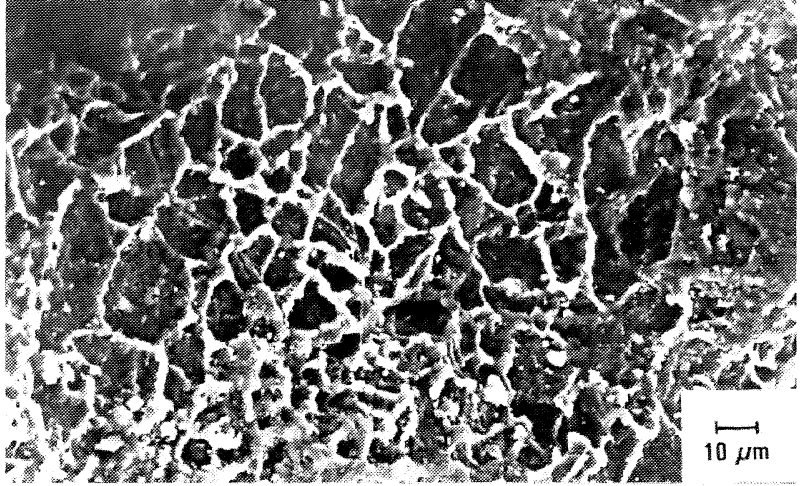

Fig. 11.- Micrografía en la que se observa un esqueleto de sílice de color claro sobre la matriz de óxido de hierro.

FIG. 11.- Micrography showing a form of clear color of silica on the iron oxide matrix.

- Las propiedades químicas, físicas y mecánicas de la fayalita $\left(2 \mathrm{FeO}-\mathrm{SiO}_{2}\right)$ dependen del contenido de hierro de ésta, por lo que el control de este metal en la escoria, tanto el procedente del mineral añadido (hematites) como el de la oxidación de la mata, debían guardar un equilibrio para no empeorar la viscosidad y los valores de temperatura de fusión de la escoria.

- La presencia de tectosilicatos (zeolitas) fácilmente fusibles, en cantidades minoritarias, permitían la mejor fluidez de la escoria y rebajar algo su punto de fusión, por lo que se piensa que su presencia no fue casual o inevitable.

\section{Agradecimiento}

Al Pueblo de Cerro Muriano, por su total apoyo en este estudio.

\section{REFERENCIAS}

(1) Sanchez Santos. J.C. Roma. La Romanización. Cuadernos del Museo Arqueológico Nacional. Madrid, 1991.

(2) Mohen, J.P. Metalurgia Prehistórica. Introducción a la Paleometalurgia. Ed. Masson, S.A. París, 1992: 71-92.

(3) Healy, J.F. Mining and Metallurgy in the Greek and Romand Word. Ed. Thames and Hudson, 1978: 30-45.

(4) Plinio, el Viejo (Naturalis Historia, X, 4, 95.

(5) Tacito (Ann. VI 19); Suetonio (Tiberio XLIX).

(6) Suetonio. Tiberio, XLIX.

(7) Calabrés, R. Criado, A.J. Martinez, J.A. y Storch de Gracia, J.J. Rev. Metal. Madrid, 31 (5), 1995: 298-306.

(8) CRIADO, A.J. Diario de Córdoba. 16 de enero de 1995.

(9) TylecotTE R.F. The early history of metallurgy in Europe. Ed. Longman. Londres y Nueva York, 1987: 329 y 102-115.

(10) Handbook of Chemistry and Physics. CRC Press, Inc. 64 th. Ed. 